\title{
Sero-prevalence of Leptospira spp. in Household and Stray Dogs by Microscopic Agglutination Test
}

\author{
Ali Shabestari As $\mathbf{l}^{1^{*}}$, Mohammad Bafandehzendeh², Gholamreza Abdollahpoor ${ }^{3}$ \\ ${ }^{1}$ Department of Clinical Sciences, Faculty of Veterinary Medicine, Tabriz Branch, Islamic Azad University, Tabriz, Iran \\ ${ }^{2}$ Young Researchers Club, Tabriz Branch, Islamic Azad University, Tabriz, Iran \\ ${ }^{3}$ Department of Clinical Sciences, Faculty of Veterinary Medicine, University of Tehran, Tehran, Iran
}

\begin{abstract}
Background: Leptospirosis is a zoonotic disease of worldwide significance in human beings and many animals. This zoonotic disease causes infection in dogs and other pet animals and these animals can influence human's health. Given the unknown prevalence of leptospirosis in dogs in our area, this study was performed to investigate the sero-epidemiology of leptospirosis and evaluate the seroprevalence of Leptospira serovars in both household and stray dogs by microscopic agglutination test (MAT). Methods: Serum samples of 90 dogs in two household and stray groups (45 dogs for each group) were collected. Specimens were examined by MAT (in accordance with the procedure recommended by WHO) against antigens of serovars Canicola, Grippotyphosa, Icterohaemorrhagiae, Pomona, Hardjo, Autumnalis, and Ballum.

Results: In total, $6.7 \%$ of $(n=6)$ dogs ( 1 stray and 5 household dogs) had a serum titer of 1:100 or more against Leptospira serovars. Dominant serovars were Canicola $(8.9 \%)$, Grippotyphosa $(2.2 \%)$, and Pomona $(2.2 \%)$, respectively. The antibody titer against more than one serovar was observed only in one dog. The sero-prevalence was $11.1 \%$ in household dogs and $2.2 \%$ in stray dogs. Since 4 pet dogs had been vaccinated recently, it seems that the actual percentage of infection in household dogs must be just $2.2 \%$ and actual infection in dogs including stray dogs is approximately $4.4 \%$ in Tabriz.

Conclusion: Fortunately, the rate of infection in Tabriz is low and this can be due to special geographical and climatic conditions, regular and annual vaccination of household dogs against this disease, and lack of suitable conditions for the growth and survival of such micro-organisms in the environment.

Keywords: Leptospirosis, Dog, MAT, Serovar
\end{abstract}

Received: December 6, 2019, Accepted: December 17, 2019, ePublished: January 1, 2020

\section{Introduction}

Leptospirosis, which is caused by spiral spirochetes, is a zoonotic disease of worldwide significance and is responsible for the infection and creation of several illnesses in human beings and many animals (1). Moreover, leptospirosis has been observed in dogs, cats, coyotes, monkeys, foxes, wolves, chipmunks, pigs, camels, sheep, goats, and cows (2). The genus Leptospira is an incredibly varied group of organisms, including hundreds of serovars and genetic types. Approximately, 250 different serovars have been identified in the Leptospira genus. Although the pathogenic importance of many serovars is unknown, 6 to 8 are thought to be pathogenic in dogs (3). Leptospira interrogans serovars Canicola, İcterohaemorrhagiae, and Leptospira kirschneri serovar Grippotyphosa are the most common serovars isolated from dogs with leptospirosis (4). Furthermore, serovars Pomona and Bratislava are the two most common serovars in the United States (5). In addition, serovars such as Australis, Javanica, Ballum, Hardjo, Autumnalis, and Pyrogenes are reported to be isolated from dogs (6).
The most common serovars reported in Iran are Hardjo, Pomona, Icterohaemorrhagiae, and grippotyphosa. A recent study shows that the prevalence of Canicola serovar among cattle has increased in different provinces (7). Some similar changes were seen in other parts of the world. For example, recent serological evidence indicated the change of dominant Leptospira serovars in dogs (8). Dogs are not the reservoir hosts for any pathogenic serovars except for Leptospira interrogans serovar Canicola. The reservoir hosts for the other serovars include rodents, skunks, raccoons, and deer (9). In Iran, the first extensive study about leptospirosis was conducted by Maghami et al in 1957 and its results indicated that $31 \%$ of cows and $17 \%$ of sheep were infected with serotypes of Grippotyphosa, Pomona, Hius, and Icterohaemorrhagiae. Recent studies which were conducted in Leptospira Laboratory of Tehran University on samples from different cities from 2002 to 2006 indicate that the serologic prevalence of the disease in both human beings and animals is between 25 to $42 \%$ (10). The sero-prevalence in Northern provinces of Iran is reported to be high in both animals and human beings 
and even in Guilan province, 35\% of rice farms, 35\% of water channels, and $30 \%$ of rivers are infected with these organisms. The same report has shown a high prevalence of Pomona in rice farms, but sarmin serogroup has been rather widespread in rivers (11).

In Iran, the first isolation of Leptospira canicola from a dog was conducted by Jamshidi et al in 2003. Jamshidi also reported a sero-prevalence of $27 \%$ in cats of Tehran in 2009 and found that the most common serovars diagnosed in stray cats were canicola and Pomona and in household cats, Hardjo, Icterohaemorrhagiae, Grippotyphosa, and Canicola were detected. Rad et al (2) reported a sero-prevalence of $31 \%$ in dogs of Tehran in 2004; however, in 2010, Khorami et al (12) reported that of 90 dogs examined, $31 \%$ shed this bacterium through urine. Avizeh et al in a study on dogs of Ahvaz reported a sero-prevalence of $4.5 \%$ (8). In a study, the rate of infection or sero-prevalence in other animals, especially in herbivores, ranged between 18 and $58 \%$ and canicola and Grippotyphosa were the most common serovars, while Hardjo, Pomona, and Icterohaemorrhagiae were less common (12).

Different studies indicate a rather high prevalence in dogs, which varies between 7 and $84 \%$. Besides different percentages of sero-positivity in different parts of the world, the percentages of different Leptospira species in dogs differ. Thailand (83.5\%) and India (62.5\%) have the highest percentages of sero-positivity in the world. The prevalence of different species varies due to geographical conditions even in one country, and reports from California and New York show discrepancies in species (13).

It seems that contact with the contaminated water is the most common method of spread. Stagnant or slowmoving warm water, not necessarily, provides a suitable environment for spirochetes and it seems that the disease outbreaks increase during the flood periods $(14,15)$. However, these organisms can be transmitted between animals by direct contact, venereal and placental transfer, bite, or ingestion of infected meat (16).

Therefore, considering the rather high infection rate in animals and its easy transmission, especially in dogs, the sero-epidemiologic study and determination of infection rate or sero-positivity as well as the examination of common serovars of each area are significant and can be helpful in effective diagnosis and treatment of infection. Due to the lack of studies about the prevalence of infection and sero-prevalence in dogs of this region, the present study can determine the seroprevalence and the type of involved species in dogs of this region.

\section{Materials and Methods}

In this study, serum samples of 45 dogs in both household and stray groups were collected randomly from May to September 2019. Household (pet) dogs were chosen from different veterinary clinics in Tabriz and also clients of Small Animal Clinic of Azad University and stray dogs were chosen from dogs that trapped by Tabriz municipality. In each group, thorough check-ups were conducted and a questionnaire for each animal concerning type of the dog (household or stray) was filled out. All of the household dogs were presented to clinics for ordinary examinations, anti-parasite treatments, or annual vaccination. In clinical examination, there was not any symptom representing any infectious disease and none of the owners had any complaints about the presence of disease or other suspicious signs of this disease. None of the stray dogs represented any signs related to clinical or infectious diseases.

For the evaluation of sero-positivity, $5 \mathrm{~mL}$ of venous blood was taken from any available vein (especially cephalic vein). After blood sampling, specimens were centrifuged at $3000 \times$ for 5 minutes and immediately after the isolation of serum, these serums were kept in sterile microtubes at $-20^{\circ} \mathrm{C}$ until use for serological examinations. The samples were sent to Leptospira Laboratory in Veterinary College of Tehran University for completing micro agglutination test (MAT).

\section{Micro Agglutination Test}

In general, this test is done in accordance with the procedure suggested by the WHO, and in this study, it was conducted in Leptosprios Laboratory in Veterinary College of Tehran University. According to the standard of $\mathrm{WHO}$, the samples with the agglutination degrees of $+1,+2,+3$, and +4 were regarded as negative, suspected, positive and serious, respectively. In the second stage, MAT was studied in serological samples with +4 agglutination degree along with titration method in more than 1:100 of serological titers.

\section{Results}

Among 90 dogs which were tested by MAT, 6.7\% $(n=6)$ had titers of 1:100 or more. Moreover, $11.1 \%$ of household dogs $(n=5)$ and $2.2 \%$ of stray dogs $(n=1)$ showed seropositivity against Leptospira antigens. The most prevalent serovar was Leptospira interrogans serovar Canicola that was observed in $8.9 \%$ of dogs $(n=4)$ and there was only one dog which had positive titer against Leptospira kirschneri serovar Grippotyphosa (2.2\%), as well as Leptospira interrogans serovar Pomona (2.2\%). Additionally, in one of the pet dogs, both Canicola and Grippotyphosa antigens were observed simultaneously.

Statistical analysis indicated no significant difference between the types of the dogs (household and stray dogs) in terms of the effects of age, gender, and breed on the amounts of MAT titers among dogs $(P>0.05)$. However, the type of keeping dogs (indoor, outdoor) caused a significant difference in antibody levels $(P<0.05)$. Considering the presence of antibody titer which resulted from the vaccination in household dogs (4 out of 32 household vaccinated dogs), it is assumed that the effect 
of vaccination on the amounts of antibody titer were not significantly different between the vaccinated and unvaccinated household dogs $(P>0.05)$.

\section{Discussion}

Leptospirosis is a zoonotic disease of worldwide significance which is observed in most of the mammals. Because of the variety of Leptospira serovars, the causal agent can be kept by many of wild and domestic animals (17). Therefore, there are different reservoir animals which have the potential source for introducing disease to human beings. Additionally, dogs and other pet animals can also transmit different serovars such as icterohaemorrhagiae and Canicola to human $(18,19)$. Therefore, considering leptospirosis as a zoonotic disease and also the possibility of infection in dogs, there is a need for studying the presence of infection or sero-positivity in different areas.

Based on animal species, climate, geographical area, and presence of different vectors and reservoirs, different infection percentages have been reported (20,21). However, because of the geographic and environmental features of Azerbaijan, the varieties of involved Leptospira serovars in dogs are somehow vague. Furthermore, the extension of disease whether in human beings and animals is an important reason in the evaluation of leptospirosis and assessment of involved serovars. Unfortunately, the increase of infection in Iranian people was reported especially in recent years $(22,23)$. For example, in a study which was conducted on 400 persons in tribal areas of West Central Iran, it was specified that $48.5 \%$ of tested ones had antibody titer against Leptospira serovars and that women had more antibody than men and the most dominant serovar was specified to be hardjo $(24,25)$. In another study conducted in 4 different provinces of Iran by Zakeri et al (26), the sero-positivity was identified to be $26.5 \%$. Therefore, considering the above-mentioned rates in different areas of Iran and the lack of awareness about the presence of these agents in the dogs of the area, it is necessary to conduct a comprehensive study for specifying the rate of occurrence and the types of Leptospira serovars in dogs.

In the present study, regarding the evaluation of seropositivity in stray dogs, it was observed that only one of them $(2.2 \%)$ had 1:100 titers which seem to be the lowest percentage of natural infection in Iran. Given the conditions of stray dogs and also the possibility of high contaminating of these dogs with vectors and reservoir animals, water, carcass, and contaminated materials, the probability of infection in these dogs are likely high. However, only $2.2 \%$ of stray dogs were infected and the involved species were identified to be $L$. interrogans serovar Pomona. Due to the lack of reasonable reports concerning Iranian dogs (either household or stray) infected with this serovar, this study seems to be the first report of serovar Pomona infection in dogs in Iran.
In the household dogs, among 45 tested dogs, 5 dogs showed 1:100 titer and more. An imported 8-month-old Siberian husky breed was one of these dogs that showed high titer (more than 1:100) without vaccination history. Therefore, considering the lack of vaccination and the presence of more than 1:100 titers in this dog, it must be regarded as infected with leptospirosis in which serovar Canicola was involved (26).

In addition to this dog, 4 pet dogs ( 1 male and 3 females, all of which were kept inside home) also had 1:100 titer and more. It can be concluded that the high serological titer was due to the use of leptospiral vaccines because all of these dogs were vaccinated against different serovars of leptospiral organisms and received their vaccinations during the last 8 months. Considering the presence of proper amount of titer, even after 1 year, in dogs which were vaccinated at least two times in their young lives (27), it can be concluded that the high serological titer resulted from regular vaccinations. Therefore, regarding the lack of symptoms of acute leptospirosis at the time of sampling and also the lack of complaint by the owner about the signs of leptospirosis, it seems that the high serological titer resulted from regular and annual vaccinations in these animals. However, for determining the exact infection rate in the mentioned dogs, urine samples should also be analyzed.

\section{Conclusion}

Considering the results of this study, it can be concluded that the true infection rate in Azerbaijan is $4.4 \%$ which is the lowest percentage of infection in Iran. On the other hand, considering the changes in the infection rate related to seasonal condition and the increase of infection after the rain in spring and summer, the percentage of infection or sero-positivity in dogs of this region seems to be very low.

However, considering the high antibody titer in other animals in the region such as cows (24\%), horses (41.05\%), and sheep (18.4\%), it seems that the time of sampling, season, climate change, amount of rain in different years, and also animal species have a well-deserved effect on the prevalence of Leptospiral organisms.

Therefore, for determining the percentage of infection in different animals in the same region, sampling from different animals must be done at the same time to determine the sensitivity of different species of animals to different serovars. It is also recommended that for determining the exact amount of infection, serum and urine samples should be analyzed simultaneously as animals are capable of shedding microorganisms in their urine.

\section{Conflict of Interests}

The authors declared that no competing interests exist.

Ethical Issues

In this research, ethical considerations have been fully observed. 


\section{Acknowledgment}

The authors would like to express their deep gratitude to managers and staff of leptospirosis Laboratory of Tehran University and Small Animal Clinic of Islamic Azad University, Tabriz Branch, for providing the research team with valuable theoretical and practical assistance in conducting the present study.

\section{Authors' Contribution}

ASA did writing and editing of the manuscript. MB designed and did data collection. GA designed, did data collection and statistical analysis.

\section{Funding}

The authors received no financial support for the research, authorship and publication of this article.

\section{References}

1. Greene CE, Emmett BS. Leptospirosis. In: Greene, CE, ed. Infection Disease of the Dogs and Cats. USA: Saunders Publication; 1990. p. 498-507.

2. Rad MA, Zeinali A, Vand Yusofi J, Tabatabayi AH, Bokaie $S$. Seroprevalence and bacteriological study of canine leptospirosis in Tehran and its suburban areas. Iran J Vet Res. 2004; 5(2):73-80.

3. Goldstein RE. Bacterial diseases (Leptospirosis). In: Ettinger SJ, Feldman EC, eds. Textbook of Veterinary Internal Medicine: Diseases of the Dog and the Cat. Canada: Elsevier Saunders; 2010. p. 863-9.

4. Goldstein RE, Lin RC, Langston CE, Scrivani PV, Erb HN, Barr SC. Influence of infecting serogroup on clinical features of leptospirosis in dogs. J Vet Intern Med. 2006;20(3):489-94. doi: 10.1892/0891-6640(2006)20[489:ioisoc]2.0.co;2.

5. Vijayanand V, Prasad AA, Shammi M, Rajasundaram RC. Serological evidence of leptospiral antibodies in dogs in Peripheral areas around Chennai. Indian J Vet Anim Sci. 2008;4(4):154-5.

6. Abdollahpour G, Shafighi T, Sattari Tabrizi S. Serodiagnosis of leptospirosis in cattle in north of Iran, Gilan. Iran J Med Sci. 2009;3(1):7-10. doi: 10.22059/ ijvm.2009.19603.

7. Abdollahpour G, Tabatabaei AM, Shafighi M, S.T., Sattari Tabrizi S. Seroprevalence of Leptospira interrogans in Cattle in Northern Iran, Gilan Province. Thailand: Proceedings of the 4th ILS-2005 Meeting; 2005. p. 315.

8. Avizeh R, Ghorbanpoor M, Hatami S, Abdollahpor G. Seroepidemiology of canine leptospirosis in Ahvaz, Iran. Iran J Med Sci. 2009;2(2):75-9. doi: 10.22059/ ijvm.2008.27369.

9. Issazadeh K, Amirmozaffari N, Mehrabian S, Oryan H. Assessment of distribution Leptospira spp. in surface waters of Guilan province. American-Eurasian Journal of Scientific Research. 2008;3:147-52.

10. Jamshidi S, Vandeussefi GM, Dezfoulian O, Selk Ghaffari M. Isolation of Leptospira canicola from a dog in Iran: first report. Iran J Vet Res. 2008;9(3):291-4. doi: 10.22099/ ijvr.2008.1754

11. Jamshidi S, Akhavizadegan MA, Bokaie S, Maazi N, Ghorban Ali A. Serologic study of feline leptospirosis in
Tehran, Iran. Iran J Microbiol. 2009;1(2):32-6.

12. Khorami N, Malmasi A, Zakeri S, Zahraei Salehi T, Abdollahpour G, Nassiri SM, et al. Screening urinalysis in dogs with urinary shedding of leptospires. Comp Clin Path. 2010;19(3):271-4. doi: 10.1007/s00580-009-0856-1.

13. Ebrahimi A, Nasr Z, Kojouri GH. Seroinvestigation of bovine leptospirosis in Shahrekord district, central Iran. Iran J Vet Res. 2004;5(2):110-3.

14. Ebrahimi A, Alijani L, Abdollahpour G. Serological survey of human leptospirosis in tribal areas of west central Iran. Iran J Med Sci. 2015;28(2):93-5.

15. Haji Hajikolaei MR, Ghorbanpour M, Abdollapour G. Seroprevalence of leptospiral infection in buffalo (Bubalus bubalis). Bull Vet Inst Pulawy. 2006;50(3):341-4.

16. Hasanpour A, Fartashvand M, Abdollahpour G, Mogaddam GH, Nadalian M, Sattari S. Seroprevalence of leptospiral infection in dairy herds in Tabriz-Iran. Pajouhesh-va-Sazandegi. 2007;19(1):6777.

17. Tooloie M, Abdollahpour G, Karimi H, Hasanpor A. Prevalence of serum antibodies against six Leptospira serovars in sheep in Tabriz, northwestern Iran. J Anim Vet Adv. 2008;7(4):450-5.

18. Hassanpour, A., Monfared, N., Abdollahpour, G.R., Satari, S., 2009. Seroprevalence of leptospiral infection in horses in Tabriz Iran. Journal of Bacteriology Research. 1, 97-100.

19. Adin CA, Cowgill LD. Treatment and outcome of dogs with leptospirosis: 36 cases (1990-1998). J Am Vet Med Assoc. 2000;216(3):371-5. doi: 10.2460/javma.2000.216.371.

20. Scanziani E, Origgi F, Giusti AM, lacchia G, Vasino A, Pirovano $\mathrm{G}$, et al. Serological survey of leptospiral infection in kennelled dogs in Italy. J Small Anim Pract. 2002;43(4):154-7. doi: 10.1111/ j.1748-5827.2002.tb00048.x.

21. Davis MA, Evermann JF, Petersen CR, VancerSchalie J, Besser TE, Huckabee J, et al. Serological survey for antibodies to Leptospira in dogs and raccoons in Washington State. Zoonoses Public Health. 2008;55(8-10):436-42. doi: 10.1111/j.1863-2378.2008.01137.x.

22. Jittapalapong S, Sittisan P, Sakpuaram T, Kabeya H, Maruyama S, Inpankaew T. Coinfection of Leptospira spp and Toxoplasma gondii among stray dogs in Bangkok, Thailand. Southeast Asian J Trop Med Public Health. 2009:40(2):247-52.

23. Okewole EA, Ayoola MO. Seroprevalence of leptospiral serovars other than Canicola and Icterohaemorrhagiae in dogs in the Southwestern Nigeria. Vet Arh. 2009;79(1):87-96.

24. Honarmand HR, Eshraghy S, Khorami Zadeh MR, Mansour Ghanaie F, Fallah MS, Rezvani M, et al. Survey spread of positive lepotospirosis by ELISA in Guilan province. Journal of Guilan University of Medical Sciences. 2005;14(54):59-67.

25. Zakeri S, Sepahian N, Afsharpad M, Esfandiari B, Ziapour P, Djadid ND. Molecular epidemiology of leptospirosis in northern Iran by nested polymerase chain reaction/restriction fragment length polymorphism and sequencing methods. Am J Trop Med Hyg. 2010;82(5):899-903. doi: 10.4269/ajtmh.2010.09-0721.

26. Zakeri S, Khorami N, Ganji ZF, Sepahian N, Malmasi AA, Gouya MM, et al. Leptospira wolffii, a potential new pathogenic Leptospira species detected in human, sheep and dog. Infect Genet Evol. 2010;10(2):273-7. doi: 10.1016/j.meegid.2010.01.001.

27. Klaasen HL, Molkenboer MJ, Vrijenhoek MP, Kaashoek MJ. Duration of immunity in dogs vaccinated against leptospirosis with a bivalent inactivated vaccine. Vet Microbiol. 2003;95(1-2):12132. doi: 10.1016/s0378-1135(03)00152-4

(C) 2020 The Author(s); This is an open-access article distributed under the terms of the Creative Commons Attribution License (http:// creativecommons.org/licenses/by/4.0), which permits unrestricted use, distribution, and reproduction in any medium, provided the original work is properly cited. 